\title{
On-shell recursion relations for generic theories
}

\author{
Clifford Cheung ${ }^{a, b}$ \\ ${ }^{a}$ Berkeley Center for Theoretical Physics, UC Berkeley, \\ Berkeley, CA 94720 U.S.A. \\ ${ }^{b}$ Theoretical Physics Group, LBNL, \\ Berkeley, CA 94720 U.S.A. \\ E-mail: clifford.cheung@berkeley.edu
}

ABSTRACT: We show that on-shell recursion relations hold for tree amplitudes in generic two derivative theories in an arbitrary number of dimensions with multiple particle species and diverse spins. For example, in a gauge theory coupled to scalars and fermions, any amplitude with at least one gluon obeys a recursion relation. In (super)gravity coupled to scalars and fermions, the same holds for any amplitude with at least one graviton. This result pertains to a broad class of theories, including QCD, $\mathcal{N}=4 \mathrm{SYM}$, and $\mathcal{N}=8$ supergravity.

KeYwords: Supersymmetric gauge theory, Gauge Symmetry, Field Theories in Higher Dimensions, Classical Theories of Gravity

ARXiv EPRINT: 0808.0504 


\section{Contents}

$\begin{array}{llr}1 & \text { Introduction } & 1\end{array}$

2 A proof of recursion relations $\quad 2$

3 Spin $\leq 1$ amplitudes $\quad 4$

3.1 Background field lagrangian 5

3.2 Eliminating $\mathcal{O}(z)$ vertices 5

$\begin{array}{lll}3.3 & \text { Checking explicit diagrams } & 7\end{array}$

3.4 Mixed gluon amplitudes 9

4 Spin $\leq 2$ amplitudes 10

$\begin{array}{lll}4.1 & \text { Background field lagrangian } & 10\end{array}$

4.2 Eliminating $\mathcal{O}\left(z^{2}\right)$ and $\mathcal{O}(z)$ vertices 11

4.3 What are $X^{a \bar{a} b \bar{b}}$ and $Y^{\lambda a \bar{a} b \bar{b}}$ ? 13

$\begin{array}{lll}4.4 & \text { Checking explicit diagrams } & 14\end{array}$

$\begin{array}{ll}\text { 4.5 Mixed graviton amplitudes } & 15\end{array}$

5 Conclusion $\quad 16$

$\begin{array}{ll}\text { A Exact expressions for } X^{\mu \nu \rho \sigma} \text { and } Y^{\lambda \mu \nu \rho \sigma} & 17\end{array}$

\section{Introduction}

There is gathering evidence that on-shell amplitudes are far simpler than one would naively expect from conventional quantum field theory. This observation goes back more than two decades, when Parke and Taylor [1] showed that tree-level maximally helicity violating (MHV) gluon amplitudes take an incredibly simple form. In recent years further progress has been made, particularly regarding amplitudes in gauge and gravity theories. Largely inspired by Witten's twistor formulation of 4D Yang-Mills [2], techniques like the CSW rules [3] and the BCFW recursion relations $[4,5]$ have provided a better theoretical understanding of gauge theories, as well as a practical toolbox of methods for calculating amplitudes. Since then, recursion relations have also been derived for gauge theories with massive particles [6] and pure gravity [7-10].

In a nutshell, the BCFW recursion relations are a way of writing on-shell tree amplitudes as a sum over products of lower point on-shell tree amplitudes evaluated at complex momenta. Calculationally, they are an incredibly efficient method for computing amplitudes since they directly relate physical amplitudes, making no reference to an underlying Lagrangian or the machinery of Feynman diagrams. Indeed, despite their usual versatility, 
Feynman diagrams simply become too cumbersome and numerous to be effective when the number of external legs becomes large. Moreover, Feynman diagrams are, arguably, a somewhat un-physical representation of multi-particle scattering since they are not even individually gauge invariant. In contrast, the recursion relations make no reference to off-shell data, and are indicative of a completely on-shell (albeit complexified) S-matrix formulation of quantum field theory.

Ultimately, recursion relations are possible because tree amplitudes are rational functions of the external momenta. Thus, given a complex deformation of the momenta parameterized by a complex number $z$, the amplitude will be a meromorphic function of $z$. Assuming furthermore that the amplitude vanishes as $z \rightarrow \infty$, then it is characterized entirely by its poles. Since each pole can be thought of as a factorization channel of the amplitude, the residue at each pole is simply a product of lower point amplitudes. The bottom line is that vanishing large $z$ behavior is a sufficient condition for the existence of recursion relations.

In this paper we argue that this criterion is satisfied by tree amplitudes in two derivative gauge and (super)gravity theories coupled to scalars and fermions. For example, in a theory of spin $\leq 1$, any amplitude with at least one gluon can be recursed, while in a theory of spin $\leq 2$, this is true of any amplitude with at least one graviton. Some notable examples for which this holds are $\mathrm{QCD}, \mathcal{N}=4 \mathrm{SYM}$ and $\mathcal{N}=8$ supergravity. Our proof follows the approach of [11], and likewise holds for an arbitrary number of dimensions.

The outline of the paper is as follows. In section 2 we review the derivation of recursion relations for a generic tree amplitude. We show that vanishing large $z$ behavior implies that an amplitude can be recursed. In sections 3 and 4 we argue that this criterion is satisfied in a broad class of amplitudes in gauge and (super)gravity theories coupled to scalars and fermions. We conclude in section 5 .

\section{A proof of recursion relations}

The BCFW recursion relations were originally proven for Yang-Mills theory in $D=4$ dimensions using the spinor helicity formalism [4, 5]. The proof was later extended to arbitrary $D$ in [11], where it was also emphasized that recursion relations are a generic property of tree-level amplitudes that vanish at large complex momenta. In this section we review these arguments, keeping the discussion general and assuming nothing about the particle content or interactions of the underlying theory.

To begin, consider a tree amplitude with $N+2$ external legs in $D$ dimensions. We label two of the legs by 1 and 2 , and their momenta by $p_{1}$ and $p_{2}$, respectively. We label the other $N$ momenta by $k_{i}$. It is possible to deform $p_{1}$ and $p_{2}$ in a complex momentum direction $q$ while still maintaining momentum conservation:

$$
\begin{aligned}
& p_{1}(z)=p_{1}+z q \\
& p_{2}(z)=p_{2}-z q
\end{aligned}
$$


where $z$ is a complex parameter. We define $q^{2}=q \cdot p_{1,2}=0$, so that $p_{1,2}(z)$ are still on-shell momenta, albeit complex momenta. Since our main concern is the large $z$ behavior of amplitudes, it is natural to refer to $p_{1,2}(z)$ as "hard" and $k_{i}$ as "soft".

Applying the Cauchy's residue theorem, we know that

$$
\oint \frac{\mathcal{M}(z)}{z}=\sum_{z_{I}} \operatorname{Res}\left(\frac{\mathcal{M}\left(z_{I}\right)}{z_{I}}\right)=0
$$

where $z_{I}$ label the poles of $\mathcal{M}(z) / z$. If $\mathcal{M}(z) \rightarrow 0$ as $z \rightarrow \infty$, then there is no pole an infinity and the sum is only over residues at finite $z_{I}$. From the pole at $z=0$ we obtain $\mathcal{M}(0)$, which we immediately recognize as the tree amplitude at real external momenta. Since tree amplitudes are rational functions of the external momenta, we know that the rest of the poles occur when a sum of external momenta go on-shell. To see this explicitly, let us partition all the soft momenta into two groups $I_{1}$ and $I_{2}$, associated with particles 1 and 2 , respectively. We assume these groups are of size $N_{1}$ and $N_{2}$, so $N_{1}+N_{2}=N$. We label the soft momenta by $k_{i_{1}}$ and $k_{i_{2}}$, where $i_{1} \in I_{1}$ and $i_{2} \in I_{2}$, and the sum of the soft momenta in each group by $K_{I_{1}}=\sum k_{i_{1}}$ and $K_{I_{2}}=\sum k_{i_{2}}$. In this language, the condition of momentum conservation becomes $p_{1}(z)+p_{2}(z)+K_{I_{1}}+K_{I_{2}}=0$. Next, consider the pole that occurs when $p_{1}(z)+K_{I_{1}}$ goes on-shell. We denote the value of $z$ at this pole by $z_{I_{1}}$. Near $z_{I_{1}}$, the amplitude factorizes into two lower point amplitudes evaluated at $z_{I_{1}}$ :

$$
\mathcal{M}^{N+2}\left(p_{1,2}(z), k_{i}\right) \rightarrow \sum_{h} \mathcal{M}^{N_{1}+2}\left(p_{1}(z), k_{i_{1}}, h\right) \frac{1}{\left(p_{1}(z)+K_{I_{1}}\right)^{2}} \mathcal{M}^{N_{2}+2}\left(p_{2}(z), k_{i_{2}},-h\right)
$$

where we have included superscripts that label the number of external legs in each amplitude. Here $h$ sums over the species and polarization of the intermediate particle going on-shell. Thus, the sum over poles is equivalently a sum over factorization channels of the amplitude, where the residue at each pole is the product of lower point amplitudes evaluated at the pole. Rewriting the sum over poles as a sum over the partitions $I_{1}$ and $I_{2}$, we obtain the recursion relation

$$
\mathcal{M}^{N+2}\left(p_{1,2}, k_{i}\right)=\sum_{I_{1}, h} \mathcal{M}^{N_{1}+2}\left(p_{1}\left(z_{I_{1}}\right), k_{i_{1}}, h\right) \frac{1}{\left(p_{1}+K_{I_{1}}\right)^{2}} \mathcal{M}^{N_{2}+2}\left(p_{2}\left(z_{I_{1}}\right), k_{i_{2}},-h\right)(2
$$

In diagrammatic form, it is
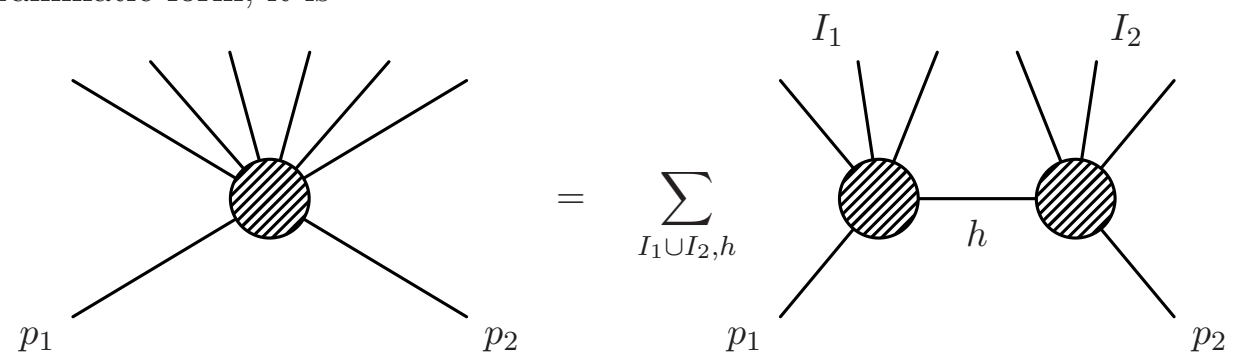

where $h$ sums over species and polarizations. We have argued that the above recursion relations hold as long as the pole at infinity is absent, i.e. as long as $\mathcal{M}(z \rightarrow \infty)=0$. Naively, one would expect this criterion to fail for gauge and gravity amplitudes, since they 
contain derivative interactions. However, in [11] it was shown that the opposite is true - an enhanced "spin Lorentz symmetry" yields vanishing large $z$ behavior in pure gauge theories and even better behavior in gravity! In this paper we show that amplitudes in generic two derivative gauge and gravity theories also satisfy the criterion that $\mathcal{M}(z \rightarrow \infty)=0$. We begin with theories of spin $\leq 1$.

\section{$3 \quad$ Spin $\leq 1$ amplitudes}

In this section we consider a generic gauge theory coupled to scalars and fermions. We argue that $\mathcal{M}(z) \rightarrow 0$ as $z \rightarrow \infty$ for any amplitude with at least one gluon. Consequently, any such amplitude obeys an on-shell recursion relation. Our reasoning is independent of any particular choice of charges for the matter fields, but requires that the theory be limited to two derivative interactions. For example, our result holds for QCD and $\mathcal{N}=4$ SYM.

Our approach follows closely that of [11]. There the authors present a particularly nice physical interpretation for the large $z$ behavior of amplitudes, which we now review. To begin, consider $\mathcal{M}(0)$, the tree amplitude at real external momenta, in the limit where $p_{1,2}$ are very hard. Taking particles 1 and 2 to be incoming and outgoing, we can interpret this process as a hard particle shooting through a haze of soft particles. In this eikonal limit, all of the soft dynamics can parameterized by a classical background through which the hard particle propagates. Thus, to determine the large momentum scaling of the amplitude, it suffices to compute the two point function of hard fluctuations in a soft background. This intuition persists in the case where $p_{1,2}$ are complexified to $p_{1,2}(z)$. Here the hard limit is defined by $z \rightarrow \infty$, but like before, all of the $z$-independent soft physics goes into determining some classical background. Thus, the bottom line is that the large $z$ behavior of an amplitude can be determined by using the background field method. Our proof occurs in three steps:

- Expand around a background: Expand the action in terms of hard fluctuations around a soft background. To evaluate $\mathcal{M}(z \rightarrow \infty)$, simply take the large $z$ limit of the two point function of fluctuations in this background.

- Choose a gauge to remove derivative interactions: Derivatives from gauge interactions naively spoil large $z$ behavior. Remove large $z$ contributions from these interactions by going to light-cone gauge for the background $(q \cdot A=0)$ and choosing an appropriate $R_{\xi}$ gauge for the fluctuation. Note that background light-cone gauge cannot be chosen for certain "unique diagrams," [11] so these diagrams must be checked explicitly.

- Check remaining diagrams: Choosing a gauge removes most derivative interactions, but we still have to check a small number of diagrams explicitly. These include i) diagrams with no hard propagators (of which the unique diagrams are a subset), and ii) diagrams with only hard fermion propagators. Because the enhanced spin Lorentz symmetry of pure gauge theory [11] persists in a gauge theory coupled to scalars and fermions, we find that these contributions all vanish at large $z$. 


\subsection{Background field lagrangian}

For concreteness, let us consider Yang-Mills theory minimally coupled to an adjoint scalar and a fundamental fermion. Our arguments will not depend on this particular choice of charges. The action is

$$
\mathcal{L}=-\frac{1}{2} \operatorname{tr} F_{\mu \nu} F^{\mu \nu}+\operatorname{tr} D_{\mu} \Phi D^{\mu} \Phi+\bar{\Psi} i \not D \Psi+\lambda \bar{\Psi} \Phi \Psi
$$

Next, let us expand around a background for every field, using lowercase/uppercase to denote fluctuations/backgrounds:

$$
\begin{aligned}
A_{\mu} & \rightarrow A_{\mu}+a_{\mu}, \\
\Psi_{\alpha} & \rightarrow \Psi_{\alpha}+\psi_{\alpha}, \\
\Phi & \rightarrow \Phi+\phi
\end{aligned}
$$

Expanding in powers of the gluon fluctuations yields

$$
\begin{aligned}
\mathcal{L} & =\mathcal{L}_{(0)}+\mathcal{L}_{(1)}+\mathcal{L}_{(2)}+\ldots \\
\mathcal{L}_{(2)} & =-\frac{1}{2} \operatorname{tr} D_{[\mu} a_{\nu]} D^{[\mu} a^{\nu]}-i \operatorname{tr}\left[a_{\mu}, a_{\nu}\right] F^{\mu \nu}-\operatorname{tr}\left[a_{\mu}, \Phi\right]\left[a_{\nu}, \Phi\right] \eta^{\mu \nu} \\
\mathcal{L}_{(1)} & =\operatorname{tr} a_{\mu} J_{(1)}^{\mu} \\
& =2 \operatorname{tr} a_{\mu}\left(\left[\Phi, i D^{\mu} \phi\right]+\left[\phi, D^{\mu} \Phi\right]\right)+\bar{\Psi} \not \phi+\bar{\psi} \not \psi
\end{aligned}
$$

where $D_{\mu}$ is a background gauge covariant derivative and $J_{(1)}^{\mu}$ is the gauge current expanded to linear order in the scalar and fermion fluctuations.

The above action only contains terms that are quadratic in the fluctuations $a, \psi$, and $\phi$. This is because we have made the very important assumption that tadpoles in the fluctuation vanish, i.e. the background fields obey their equations of motion. In terms of amplitudes this corresponds to putting soft external legs on-shell, which is of course necessary if on-shell recursion relations are to hold.

\subsection{Eliminating $\mathcal{O}(z)$ vertices}

In order to determine $\mathcal{M}(z)$, we simply compute the two point function of hard fluctuations in the presence of the soft background. We then take the large $z$ limit. Since we are concerned with large $z$ behavior, our first worry is interactions with derivatives acting on the hard fields, $a, \psi$ and $\phi$. Naively, these derivatives generate powers of $z$ that blow up as $z \rightarrow \infty$. Since these terms only show up in $D_{[\mu} a_{\nu]} D^{[\mu} a^{\nu]}, D_{\mu} \phi D^{\mu} \phi$, and the mixing term, $a_{\mu}\left[\Phi, i D^{\mu} \phi\right]$, the dangerous terms are all proportional to either $q \cdot A$ or $q \cdot a$. This statement actually holds for any two derivative theory, renormalizable or not.

Terms with $q$ dotted into the background gluon can be eliminated by choosing background light-cone gauge, that is $q \cdot A=0 .{ }^{1}$ However, as shown in [11], it is actually

\footnotetext{
${ }^{1}$ In higher derivative theories, for example Euler-Heisenberg theory, there are additional $\mathcal{O}(z)$ terms of the form $q_{\mu} F^{\mu \nu}$ that cannot be removed by background light-cone gauge. For this reason we restrict to two derivative theories.
} 


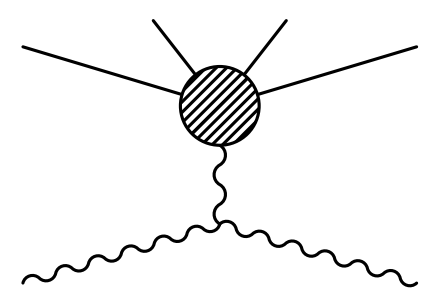

Figure 1. The unique diagram. Since gluons 1 and 2 meet directly at a vertex, the momentum flowing into the soft gluon is $p_{1}+p_{2}$. For this reason, background field light-cone gauge cannot be chosen for this diagram.

impossible to choose this particular gauge for a class of so-called unique diagrams. To see this, consider the gauge choice $A_{\mu} \rightarrow A_{\mu}^{\prime}$, where $q \cdot A^{\prime}=0$ and

$$
A_{\mu}^{\prime}=A_{\mu}+p_{\mu} \Omega
$$

Clearly, $\Omega$ becomes singular if $q \cdot p=0$, which only happens if $p=p_{1}+p_{2}$, corresponding to the unique diagram (see figure 1 ) in which particles 1 and 2 meet directly at a trilinear vertex with a soft gluon. Since we are only concerned with diagrams in which particle 1 is a gluon, the only unique diagram occurs when particle 2 is also a gluon, simply because there is no interaction between two gluons and a non-gluon. ${ }^{2}$ This unique diagram arises in pure gauge theory, and was shown to vanish at large $z$ [11].

Next, let us consider terms involving $q$ dotted into the fluctuation. These terms arise from $\left(D_{\mu} a^{\mu}\right)^{2}$ and the mixing term. We recognize the latter as simply the mixing term between gauge bosons and goldstone bosons in a gauge theory with spontaneous symmetry breaking. Consequently, both terms can be removed in the usual way by an appropriate $R_{\xi}$ gauge choice for the fluctuation:

$$
\mathcal{L}_{\xi}=\frac{1}{\xi} \operatorname{tr}\left(D_{\mu} a^{\mu}+i \xi[\Phi, \phi]\right)^{2}
$$

For the choice of $\xi=1$, all derivative interactions are removed, and the quadratic gluon action becomes

$$
\mathcal{L}_{(2)}=-\frac{1}{2} \operatorname{tr} \eta^{a b} D_{\mu} a_{a} D^{\mu} a_{b}-i \operatorname{tr}\left[a_{a}, a_{b}\right] F^{a b}-\operatorname{tr}\left[a_{a}, \Phi\right]\left[a_{b}, \Phi\right] \eta^{a b}
$$

where we have re-written some Greek indices as Latin indices in order to emphasize the spin Lorentz symmetry, which is nothing more than the fact that the kinetic term enjoys an enhanced Lorentz symmetry that acts only on the $a b$ indices. In the $z \rightarrow \infty$ limit, this term dominates over everything, so the spin Lorentz symmetry is a symmetry of the leading order in $z$ contribution. As discussed in [11], this spin Lorentz symmetry is necessary to show that pure gauge theory amplitudes vanish at large $z$.

\footnotetext{
${ }^{2}$ This is actually not true for all two derivative theories. In the presence of an axion or a singlet scalar, there can be operators of the form $a F_{\mu \nu} \tilde{F}^{\mu \nu}$ and $b F_{\mu \nu} F^{\mu \nu}$, which introduce new unique diagrams. We assume that such interactions are absent.
} 

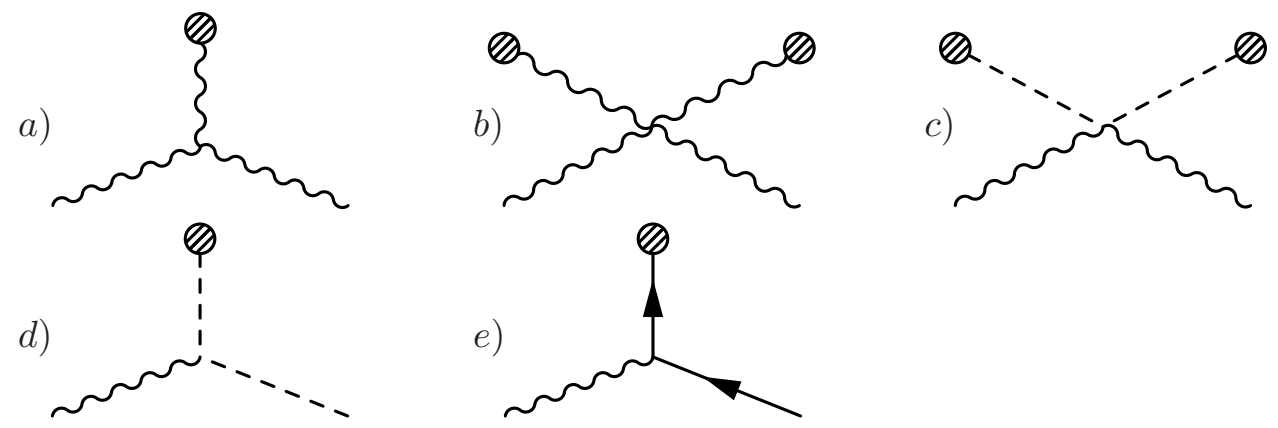

Figure 2. All diagrams with no hard propagators. The blobs represent insertions of a classical background that parameterizes all of the soft physics. In diagrams $a$ ) $-c$ ) particles 1 and 2 are both gluons, while $d$ ) and $e$ ) are mixed diagrams. Diagrams $a$ ) (the unique diagram) and $b$ ) occur in pure gauge theory; after dotting into the appropriate polarizations, they vanish at large $z$ [11]. Diagram $c$ ) is proportional to $\eta^{a b}$, so it preserves the spin Lorentz symmetry and is $\mathcal{O}(1 / z)$ after dotting into polarizations. Diagrams $d$ ) and $e$ ) are $\mathcal{O}(1 / z)$ after dotting into polarizations.

\subsection{Checking explicit diagrams}

Any Feynman diagram is simply a product of interaction vertices and propagators. Since our choice of gauge has fixed every interaction to go as $\mathcal{O}(1)$, the only question is how propagators scale at large $z$. A hard boson propagator goes as $1 /(p+z q)^{2}=\mathcal{O}(1 / z)$, while a hard fermion propagator actually scales as $\mathcal{O}(1)$ :

$$
\frac{1}{\not p(z)}=\frac{\not p+z \phi}{p^{2}+2 z p \cdot q} \stackrel{z \rightarrow \infty}{=} \frac{\not q}{2 p \cdot q}
$$

Thus, any diagram with at least one hard boson propagator will necessarily vanish at large $z$. In contrast, i) any diagram with no hard propagators, and ii) any diagram with only hard fermion propagators will naively go as $\mathcal{O}(1)$ at large $z$. In this section we explicitly check that these two classes of diagrams do not spoil the large $z$ behavior.

To begin, we assume that particles 1 and 2 are both gluons, leaving the mixed case for a later section. First, let us consider all diagrams with no hard propagators (see figures 2a-2c), all of which have the structure that particles 1 and 2 meet directly at a vertex. Consequently, these diagrams only contain one interaction vertex involving hard fields, and it can be read directly off the quadratic gluon action in equation (3.6). Since the action differs from that of a pure gauge theory simply by $\operatorname{tr}\left[a_{a}, \Phi\right]\left[a_{b}, \Phi\right] \eta^{a b}$, the two point function of hard gluons gets an additional contribution

$$
\delta \mathcal{M}_{1}^{a b}=A \eta^{a b}
$$

on top of the pure gauge theory, where here $A$ parameterizes the soft physics of the scalar background.

Next, consider the contribution from diagrams with only hard fermion propagators. Since our gauge choice leaves only $\mathcal{O}(1)$ interactions and hard fermion propagators go as $\mathcal{O}(1)$, these diagrams naively contribute at $\mathcal{O}(1)$. However, this leading order piece actually 
vanishes! To see this, we observe that the leading order in $z$ contribution is obtained by taking a $\not$ from every hard propagator numerator. Excluding even one $\not$ introduces a factor of $1 / z$, yielding a subleading contribution. For this reason, any helicity flipping insertions, such as masses or Yukawas, contribute only at $\mathcal{O}(1 / z)$.

Consequently, for the leading order contribution, the only allowed interactions along the fermion line are gauge interactions. Thus, the corresponding Feynman diagram is comprised of alternating insertions of gluons and $\not d$ terms (see figure 3 ):

$$
\begin{aligned}
\mathcal{M}^{a c_{1} \ldots c_{n} b} & \sim \gamma^{a} d \gamma^{c_{1}} d x \ldots d \gamma^{c_{n}} d \gamma^{b} \\
& \sim\left(q^{c_{1}} \ldots q^{c_{n}}\right) \gamma^{a} d \gamma^{b}
\end{aligned}
$$

where here $a$ and $b$ label the hard gluons at either end of the fermion line, and in the second line we have anti-commuted gamma matrices and used that $\not \phi k=q^{2}=0$. Without loss of generality we can split $\mathcal{M}^{a c_{1} \ldots c_{n} b}$ into components that are symmetric and anti-symmetric in $a$ and $b$. The symmetric piece is proportional to $\gamma^{(a} d \gamma^{b)}=2 q^{(a} \gamma^{b)}-2 \eta^{a b} \not$. Finally, after dotting the $c_{i}$ into soft gluon polarization vectors and sandwiching the whole expression between soft fermion polarization spinors, we obtain the contribution to the two point function of hard gluons:

$$
\delta \mathcal{M}_{2}^{a b}=B^{[a b]}+q^{(a} C^{b)}-\eta^{a b}(q \cdot C)
$$

where $B^{[a b]}$ and $C^{a}$ are functions of the soft backgrounds and $B^{[a b]}$ is anti-symmetric.

Summing the contributions from diagrams with no hard propagators and diagrams with only hard fermion propagators, we find that the full amplitude becomes

$$
\begin{aligned}
\mathcal{M}^{a b} & =\mathcal{M}_{\text {gluon }}^{a b}+\delta \mathcal{M}_{1}^{a b}+\delta \mathcal{M}_{2}^{a b} \\
& =\mathcal{M}_{\text {gluon }}^{a b}+A \eta^{a b}+B^{[a b]}+q^{(a} C^{b)}-\eta^{a b}(q \cdot C)+\mathcal{O}(1 / z)
\end{aligned}
$$

where $\mathcal{M}_{\text {gluon }}^{a b}$ is the contribution from the pure gauge theory, which was calculated in [11].

Dotting into polarizations, the amplitude becomes $\mathcal{M}=\epsilon_{1 a}^{-} \mathcal{M}^{a b} \epsilon_{2 b}$, where without loss of generality we have defined gluon 1 to have negative helicity and gluon 2 to be arbitrary. In $D=4$ dimensions, $q$ is basically the same as the polarization vectors for the real momenta $p_{1}$ and $p_{2}$; in particular, $\epsilon_{1}^{-}=\epsilon_{2}^{+}=q$ and $\epsilon_{1}^{+}=\epsilon_{2}^{-}=q^{*}$. This of course makes sense because $\epsilon_{1,2}$ and $q$ obey the same defining equations, $q^{2}=q \cdot p_{1,2}=0$. If we now complex deform $p_{1,2} \rightarrow p_{1,2}(z)$, then the polarizations must be modified appropriately to remain normalized to unity and orthogonal to $p_{1,2}(z)$. Given these constraints the polarizations take the form [11]

$$
\begin{aligned}
& \epsilon_{1 a}^{-}=q_{a} \stackrel{\text { gauge }}{=}-\frac{p_{1 a}}{z} \\
& \epsilon_{2 a}^{ \pm}=\left\{\begin{array}{cc}
q_{a}, & (+) \\
q_{a}^{*}+z p_{1 a}, & (-)
\end{array}\right.
\end{aligned}
$$

where $\epsilon_{1}^{-}=q$ is gauge equivalent to $\epsilon_{1}^{-}=-p_{1} / z$ because they are related by a gauge transformation:

$$
\epsilon_{1 \mu}^{-} \rightarrow \epsilon_{1 \mu}^{-}+p_{1 \mu}(z)\left(-\frac{1}{z}\right)
$$




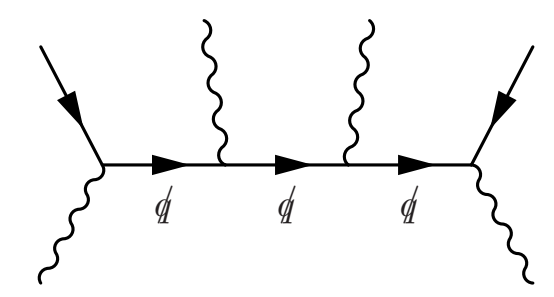

Figure 3. An example of a diagram with only hard fermion propagators. Naively, the leading $z$ contribution goes as $\mathcal{O}(1)$ and comes from taking a $q$ from every propagator numerator. However, after dotting into the external polarization for particle 1 , we find that every such diagram vanishes.

where the gauge transformation of course involves the complexified momentum. We note that in $D>4$ dimensions, there are an additional $D-4 z$-independent polarizations $\epsilon^{T}$ which span the vector space orthogonal to $p_{1,2}, q$ and $q^{*}$.

By dotting polarizations into $\mathcal{M}^{a b}$, we find that the $(-,+),(-,-)$, and $(-, T)$ amplitudes are

$$
\begin{aligned}
\mathcal{M}^{-,+} & =q_{a} \mathcal{M}^{a b} q_{b} \\
& =q_{a}\left(A \eta^{a b}+B^{[a b]}+q^{(a} C^{b)}-\eta^{a b}(q \cdot C)\right) q_{b}+\mathcal{O}(1 / z) \\
& \rightarrow \mathcal{O}(1 / z) \\
\mathcal{M}^{-,-} & =-\frac{1}{z} p_{1 a} \mathcal{M}^{a b}\left(q_{b}^{*}+z p_{1 b}\right) \\
& =-p_{1 a}\left(A \eta^{a b}+B^{[a b]}+q^{(a} C^{b)}-\eta^{a b}(q \cdot C)\right) p_{1 b}+\mathcal{O}(1 / z) \\
& \rightarrow \mathcal{O}(1 / z) \\
\mathcal{M}^{-, T} & =-\frac{1}{z} p_{1 a} \mathcal{M}^{a b} \epsilon_{b}^{T} \\
& \rightarrow \mathcal{O}(1 / z)
\end{aligned}
$$

where we have used the result from [11] to throw out the contribution from the pure gauge theory, $\mathcal{M}_{\text {gluon }}$. By convention gluon 1 can always be chosen to have negative helicity, so $\mathcal{M}^{-,+}, \mathcal{M}^{-,-}$, and $\mathcal{M}^{-, T}$ characterize any amplitude with two gluons. Since this amplitude vanishes at large $z$, the recursion relations hold.

\subsection{Mixed gluon amplitudes}

So far we have shown that recursion relations exist for amplitudes with at least two external gluons. As it turns out, this statement actually holds more generally, in particular for amplitudes with only a single external gluon. To see this, consider an amplitude where particle 1 is a gluon and particle 2 is a scalar or fermion. As before, we can fix all of the interactions to be $\mathcal{O}(1)$ using background field light-cone gauge and the appropriate $R_{\xi}$ gauge. However, since particle 2 is no longer a gluon, the explicit diagrams that we must check are now different.

First, let us consider all diagrams with only hard fermion propagators. As before, the leading order in $z$ contribution comes from taking a $\not$ from every fermion propagator 
numerator. No matter whether particle 2 is a scalar or a fermion, these diagrams take the form

$$
\mathcal{M}^{a \cdots} \sim \gamma^{a} \not 1 \ldots
$$

where the hard gluon polarization is dotted into $a$. This is always the case because particle 1 has to connect to two fermion lines, and since it is a gluon this coupling has to be a gauge interaction. Since we can choose the gluon to have negative helicity, $\epsilon_{a}^{-}=q_{a}$, this leading order contribution vanishes. Thus, the contribution from diagrams with only hard fermion propagators starts at $\mathcal{O}(1 / z)$.

This leaves diagrams with no hard propagators (see figures $2 \mathrm{~d}$ and $2 \mathrm{e}$ ). Both mixed diagrams arise from the $\mathcal{L}_{(1)}=\operatorname{tr} a_{\mu} J_{(1)}^{\mu}$, which naively contains terms that mix the hard gluon with the derivative of a hard scalar or a hard fermion. However, our choice of $R_{\xi}$ gauge eliminates these terms and so the diagrams go only as $\mathcal{O}(1)$. If we then dot the diagrams into the gluon polarization, $\epsilon_{a}^{-}=-p_{a} / z$, we find that the diagram vanishes if particle 2 is a scalar (since it has no polarization to introduce any additional factors of $z$ ), but if particle 2 is a fermion, the diagram can still go as $\mathcal{O}(1)$. An explicit check of this fermion diagram is straightforward, since it is simply a single vertex Feynman diagram. After dotting into polarizations, we find that the leading contribution goes as $\mathcal{O}(1 / z)$, for any fermion polarization. Thus, we have shown that $\mathcal{M}(z \rightarrow \infty)=0$ even when particle 2 is scalar or fermion. This completes our proof that any amplitude with at least one gluon obeys a recursion relation.

\section{Spin $\leq 2$ amplitudes}

In this section we consider a generic theory of gravity coupled to spin $0, \frac{1}{2}, 1, \frac{3}{2}$ fields. Our procedure will mirror that of the spin $\leq 1$ case. We find that $\mathcal{M}(z \rightarrow \infty)=0$ for any amplitude with at least one graviton, so this amplitude obeys a recursion relation. Our result holds for $(\mathcal{N}=8)$ supergravity.

\subsection{Background field lagrangian}

Consider (super)gravity coupled to a two derivative theory of matter ( $\operatorname{spin} \leq 1)$ fields:

$$
\mathcal{L}=\sqrt{-g} R+\mathcal{L}_{\text {matt }}\left(\Phi, \Psi_{\alpha}, A_{\mu}, \Lambda_{\mu \alpha}, g_{\mu \nu}\right)
$$

We expand the action in terms of hard fluctuations around a soft background

$$
\begin{aligned}
g_{\mu \nu} & \rightarrow g_{\mu \nu}+h_{\mu \nu} \\
\Lambda_{\mu \alpha} & \rightarrow \Lambda_{\mu \alpha}+\lambda_{\mu \alpha} \\
A_{\mu} & \rightarrow A_{\mu}+a_{\mu} \\
\Psi_{\alpha} & \rightarrow \Psi_{\alpha}+\psi_{\alpha} \\
\Phi & \rightarrow \Phi+\phi
\end{aligned}
$$


In powers of the graviton fluctuation, the action becomes

$$
\begin{aligned}
\mathcal{L}=\mathcal{L}_{(0)}+ & \mathcal{L}_{(1)}+\mathcal{L}_{(2)}+\ldots \\
\mathcal{L}_{(2)}=\sqrt{-g}( & \frac{1}{4} \nabla_{\rho} h_{\mu \nu} \nabla^{\rho} h^{\mu \nu}-\frac{1}{4} \nabla_{\mu} h \nabla^{\mu} h+\frac{1}{2} \nabla_{\mu} h \nabla_{\nu} h^{\mu \nu}-\frac{1}{2} \nabla_{\rho} h_{\mu \nu} \nabla^{\mu} h^{\nu \rho} \\
& \left.\quad+\frac{1}{2} h_{\mu \nu} h_{\rho \sigma} X^{\mu \nu \rho \sigma}+\nabla_{\lambda} h_{\mu \nu} h_{\rho \sigma} Y^{\lambda \mu \nu \rho \sigma}\right) \\
\mathcal{L}_{(1)}=\sqrt{-g}( & \left.\frac{1}{2} h_{\mu \nu} T_{(1)}^{\mu \nu}\right)
\end{aligned}
$$

Here $X^{\mu \nu \rho \sigma}$ and $Y^{\lambda \mu \nu \rho \sigma}$ are functions of the graviton and matter backgrounds and $T_{(1)}^{\mu \nu}$ is the stress-energy tensor expanded to linear order in the matter fluctuations. For now we will ignore the precise form of $X^{\mu \nu \rho \sigma}$ and $Y^{\lambda \mu \nu \rho \sigma}$, but return to them in a later section. Also, we have assumed that the background fields obey their equations of motion, so the fluctuations do not have tadpoles.

\subsection{Eliminating $\mathcal{O}\left(z^{2}\right)$ and $\mathcal{O}(z)$ vertices}

As in the gauge theory case, our first concern will be interactions that involve derivatives acting on the hard fields. In particular, we have to worry about mixing terms between the graviton and the derivatives of matter fields, which arise from $\mathcal{L}_{(1)}$. However, these dangerous contributions can always be eliminated by an appropriate $R_{\xi}$ gauge. For example, consider a free scalar coupled to gravity:

$$
\begin{aligned}
\mathcal{L}_{(1)}=\sqrt{-g}\left(\frac{1}{2} h_{\mu \nu} T_{(1)}^{\mu \nu}\right) & =\sqrt{-g} h_{\mu \nu}\left(g^{\mu \rho} g^{\nu \sigma}-\frac{1}{2} g^{\mu \nu} g^{\rho \sigma}\right) \nabla_{\rho} \phi \nabla_{\sigma} \Phi \\
& \stackrel{I b P}{=}-\sqrt{-g}\left(\nabla^{\mu} h_{\mu \nu}-\frac{1}{2} \nabla_{\nu} h\right) \phi \nabla^{\nu} \Phi+\ldots
\end{aligned}
$$

Since this term includes a derivative acting on a hard field, it will naively introduce $z$ 's into amplitudes. However, if we choose a (deDonder) $R_{\xi}$ gauge term [12]

$$
\mathcal{L}_{\xi}=\frac{\sqrt{-g}}{2 \xi}\left(\nabla^{\mu} h_{\mu \nu}-\frac{1}{2} \nabla_{\nu} h+\xi \phi \nabla_{\nu} \Phi\right)^{2}
$$

which for $\xi=1$ eliminates these dangerous terms. Since this gauge choice is simply unitary gauge for the graviton, we know that it will still work if there are also fermions and gluons in the theory. After sending $\mathcal{L} \rightarrow \mathcal{L}+\mathcal{L}_{\xi}$, we find that the quadratic graviton action becomes

$$
\mathcal{L}_{(2)}=\sqrt{-g}\left(\frac{1}{4} \nabla_{\rho} h_{\mu \nu} \nabla^{\rho} h^{\mu \nu}-\frac{1}{8} \nabla_{\mu} h \nabla^{\mu} h+\frac{1}{2} h_{\mu \nu} h_{\rho \sigma} X^{\mu \nu \rho \sigma}+\nabla_{\lambda} h_{\mu \nu} h_{\rho \sigma} Y^{\lambda \mu \nu \rho \sigma}\right)(4
$$

As written, the above Lagrangian does not have a manifest spin Lorentz symmetry. However, this can be rectified using a trick from [13], whereby a dilaton $\chi$ is introduced simply to remove the $\nabla_{\mu} h \nabla^{\mu} h$ kinetic term. Then, we perform a field redefinition

$$
h_{\mu \nu} \rightarrow h_{\mu \nu}+g_{\mu \nu} \sqrt{\frac{2}{D-2}} \chi, \quad \chi \rightarrow \frac{1}{2} g^{\mu \nu} h_{\mu \nu}+\sqrt{\frac{D-2}{2}} \chi
$$


Because of how the dilaton couples to matter, this field redefinition effectively eliminates any coupling between matter and the trace of the graviton, $h$. Moreover, since dilaton number is conserved, the dilaton completely decouples from any tree-level Feynman diagram that does not have external dilaton legs. We will be concerned only with such diagrams. After the field redefinition the quadratic graviton action takes the form

$$
\mathcal{L}_{(2)}=\sqrt{-g}\left(\frac{1}{4} \nabla_{\rho} h_{\mu \nu} \nabla^{\rho} h^{\mu \nu}+\frac{1}{2} h_{\mu \nu} h_{\rho \sigma} X^{\mu \nu \rho \sigma}+\nabla_{\lambda} h_{\mu \nu} h_{\rho \sigma} Y^{\lambda \mu \nu \rho \sigma}\right)
$$

The combination of the $R_{\xi}$ gauge and the field redefinition now makes the spin Lorentz symmetry manifest. To see this, let us rewrite the action in terms of a left and right vierbein, $e$ and $\bar{e}$ and a left and right connection, $\omega$ and $\bar{\omega}$ :

$$
\begin{aligned}
g_{\mu \nu} & =e_{\mu}^{a} e_{\nu}^{b} \eta_{a b}=\bar{e}_{\mu}^{\bar{a}} \bar{e}_{\nu}^{\bar{b}} \eta_{\bar{a} \bar{b}} \\
h_{\mu \nu} & =e_{\mu}^{a} \bar{e}_{\nu}^{\bar{a}} h_{a \bar{a}} \\
\nabla_{\rho} h_{\mu \nu} & =e_{\mu}^{a} \bar{e}_{\nu}^{\bar{a}} \nabla_{\rho} h_{a \bar{a}} \\
\nabla_{\rho} h_{a \bar{a}} & =\partial_{\rho} h_{a \bar{a}}+\omega_{\rho}^{b}{ }_{a} h_{b \bar{a}}+\bar{\omega}_{\rho}{ }^{\bar{b}} h_{a \bar{b}}
\end{aligned}
$$

Of course there is still only a single diffeomorphism redundancy, and the left/right distinction is introduced only to emphasize that there are two copies of the spin Lorentz symmetry acting on the barred and unbarred indices of the graviton. We can see this because the action now takes the form

$$
\mathcal{L}_{(2)}=\sqrt{-g}\left(\frac{1}{4} g^{\lambda \kappa} \eta^{a b} \eta^{\bar{a} \bar{b}} \nabla_{\lambda} h_{a \bar{a}} \nabla_{\kappa} h_{b \bar{b}}+\frac{1}{2} h_{a \bar{a}} h_{b \bar{b}} X^{a \bar{a} b \bar{b}}+\nabla_{\lambda} h_{a \bar{a}} h_{b \bar{b}} Y^{\lambda a \bar{a} b \bar{b}}\right)
$$

At high energies, the leading contribution is proportional to $\eta^{a b} \eta^{\bar{a} \bar{b}}$, which enjoys a double spin Lorentz symmetry.

Next, let us choose light-cone gauge for the background graviton field, i.e. for the metric $g_{\mu \nu}$. This will eliminate large $z$ contributions coming from derivatives acting on the background. As in [11], we can choose a gauge in which $q_{\mu}$ is always in the direction of the negative helicity polarization, so

$$
\begin{aligned}
& g^{--}=g^{-i}=\omega_{a b}^{-}=\bar{\omega}_{\bar{a} \bar{b}}^{-}=0 \\
& g^{-+}=1
\end{aligned}
$$

where $i$ labels all directions orthogonal to the \pm polarizations, that is, the $p_{1,2}$ and $\epsilon^{T}$ directions. This gauge choice ensures that $q$ is the same as the negative helicity polarization vector in the local Lorentz frame, i.e. $q^{2}=q \cdot p_{1,2}=q \cdot \epsilon^{T}=0$, and $q \cdot q^{*}=1$, where contractions are with respect $\eta^{a b}=\eta^{\bar{a} \bar{b}}$. This background light-cone gauge eliminates all large $z$ contributions from derivative interactions except in the unique diagrams, which were shown to vanish in [11].

Just as in the gauge case, any diagram with at least one boson propagator will get an additional factor of $1 / z$, and thus will vanish at large $z$. All that is left to check is diagrams with no hard propagators, and diagrams with only hard fermion propagators. 


\subsection{What are $X^{a \bar{a} b \bar{b}}$ and $Y^{\lambda a \bar{a} b \bar{b}}$ ?}

Before checking the remaining diagrams, it will be useful to first determine $X^{a \bar{a} b \bar{b}}$ and $Y^{\lambda a \bar{a} b \bar{b}}$. To begin, one might ask why higher derivative terms like $\nabla_{\lambda} \nabla_{\kappa} h_{a \bar{a}} h_{b \bar{b}}$ are absent from the graviton action. To see this, observe that the graviton enters $\mathcal{L}_{\text {matt }}$ only through the metric (contributing to $X^{a \bar{a} b \bar{b}}$ ), while derivatives of the graviton enter only through covariant derivatives (contributing to $Y^{\lambda a \bar{a} b \bar{b}}$ ). Since we assume a two derivative action, the only covariant derivatives come from the kinetic terms. For spin 0 and $1, \partial_{\mu} \phi=\nabla_{\mu} \phi$ and $\nabla_{[\mu} a_{\nu]}=\partial_{[\mu} a_{\nu]}$, so the covariant derivatives are simply partial derivatives and thus bosons do not contribute to $Y^{\lambda a \bar{a} b \bar{b}}$ ! For spin $\frac{1}{2}$ and $\frac{3}{2}$, there is a single covariant derivative which introduces a derivative acting on the graviton: thus, $Y^{\lambda a \bar{a} b \bar{b}}$ gets contributions from the fermion kinetic terms and $X^{a \bar{a} b \bar{b}}$ gets contributions from everything else.

It is possible to deduce the form of $X^{a \bar{a} b \bar{b}}$ and $Y^{\lambda a \bar{a} b \bar{b}}$ simply by combining spurions made up from the background fields. Let us begin with $X^{a \bar{a} b \bar{b}}$, which has a manifest $a \bar{a} \leftrightarrow b \bar{b}$ symmetry because it couples to $h_{a \bar{a}} h_{b \bar{b}}$. Since $X^{a \bar{a} b \bar{b}}$ has indices, any background field spurion from which it is constructed must also have indices. These spurions come from the kinetic terms:

$$
\partial^{a} \Phi \partial^{b} \Phi, \quad \bar{\Psi} \gamma^{a} \partial^{b} \Psi, \quad F^{a b} F^{c d}, \quad \bar{\Lambda}^{a} \gamma^{b c d} \partial^{e} \Lambda^{f}, \quad R^{a b c d}
$$

where we have not specified whether indices are left or right. Next, we simply combine these spurions with the metric in order to obtain a four index tensor. For example, since the spin 0 and $\frac{1}{2}$ spurions only have two indices, we simply multiply them by $\eta^{a b}$ or $\eta^{\bar{a} \bar{b}}$. Thus, the scalar contributes the four index tensors $\eta^{a b} \partial^{\bar{a}} \Phi \partial^{\bar{b}} \Phi$ and $\eta^{\bar{a} \bar{b}} \partial^{a} \Phi \partial^{b} \Phi$. We might in principle have multiplied by $\eta^{a \bar{a}}$, but the field redefinition in equation (4.7) eliminates any couplings of matter to the trace of the graviton, so this contribution vanishes. On the other hand, the spin 1 and 2 spurions have exactly four indices, so they contribute $F^{a b} F^{\bar{a} \bar{b}}$ and $R^{a b \bar{a} \bar{b}}$. Lastly, the spin $\frac{3}{2}$ spurion has six indices and so must be contracted with a metric to yield a four index tensor. Any single contraction will leave at least two antisymmetric indices. Since there is a $a \bar{a} \leftrightarrow b \bar{b}$ symmetry, this contribution is anti-symmetric in both $a b$ and $\bar{a} \bar{b}$. Consequently $X^{a \bar{a} b \bar{b}}$ is of the form:

$$
X^{a \bar{a} b \bar{b}}=A^{(a b)} \eta^{\bar{a} \bar{b}}+\bar{A}^{(\bar{a} \bar{b})} \eta^{a b}+B^{[a b][\bar{a} \bar{b}]}
$$

where $B^{[a b][\bar{a} \bar{b}]}$ has the symmetries of the Riemann tensor.

In contrast, $Y^{\lambda a \bar{a} b \bar{b}}$ only receives contributions from covariant derivatives in the fermion kinetic terms. This is because $\nabla_{\mu}=\partial_{\mu}+\omega_{\mu a b} \gamma^{a b} / 8$, and when linearized $\omega_{\mu a b}$ contains the derivative of the graviton. Moreover, without loss of generality we can take $Y^{\lambda a \bar{a} b \bar{b}}$ to be odd under $a \bar{a} \leftrightarrow b \bar{b}$ because the even part can always be integrated by parts and absorbed into $X^{a \bar{a} b \bar{b}}$. Before linearizing, $\omega_{\mu a b}$ enters the action as:

$$
\mathcal{L} \supset i \omega_{\mu a b}\left(\bar{\Psi} \gamma^{\mu} \gamma^{a b} \Psi+\frac{1}{12} \bar{\Lambda}_{\rho} \gamma^{\rho \sigma \mu} \gamma^{a b} \Lambda_{\sigma}\right)
$$

$Y^{\lambda a b c d}$ has to be constructed from a spurion with the same tensor structure as the quantity in parentheses. Let us call this spurion $C^{\mu a b}$, where the $a b$ indices are anti-symmetrized. 
Since $Y^{\lambda a \bar{a} b \bar{b}}$ is odd under $a \bar{a} \leftrightarrow b \bar{b}$, anti-symmetry in $a b$ implies symmetry in $\bar{a} \bar{b}$ and vice versa. Thus, in order to construct a four index tensor with the right symmetry properties, we multiply the spurion by $\eta^{a b}$ or $\eta^{\bar{a} \bar{b}}$. Thus $Y^{\lambda a \bar{a} b \bar{b}}$ is

$$
Y^{\lambda a \bar{a} b \bar{b}}=C^{\lambda[a b]} \eta^{\bar{a} \bar{b}}+\bar{C}^{\lambda[\bar{a} \bar{b}]} \eta^{a b}
$$

To see some explicit formulae for $X^{a \bar{a} b \bar{b}}$ and $Y^{\lambda a \bar{a} b \bar{b}}$, see appendix A. That said, only the generic structure shown above will be necessary for showing vanishing large $z$ behavior.

\subsection{Checking explicit diagrams}

In this section we show that all diagrams with no hard propagators and all diagrams with only hard fermion propagators vanish at large $z$. For now, consider the case where particles 1 and 2 are both gravitons.

Diagrams with no hard propagators contain only one interaction vertex involving hard momenta, so their contribution to the amplitude can be read directly off the action:

$$
\begin{aligned}
\delta \mathcal{M}_{1}^{a \bar{a} b \bar{b}} & =X^{a \bar{a} b \bar{b}}+\left(p_{1}+z q\right)_{\lambda} Y^{\lambda a \bar{a} b \bar{b}} \\
& =A^{a b} \eta^{\bar{a} \bar{b}}+\bar{A}^{\bar{a} \bar{b}} \eta^{a b}+B^{[a b][\bar{a} \bar{b}]}+z\left(C^{[a b]} \eta^{\bar{a} \bar{b}}+\bar{C}^{[\bar{a} \bar{b}]} \eta^{a b}\right)
\end{aligned}
$$

where $A^{a b}=A^{(a b)}+p_{1 \lambda} C^{\lambda a b}$ and $q_{\lambda} C^{\lambda a b}=C^{a b}$ and the same for the barred variables. It is interesting to note that $\delta \mathcal{M}_{1}^{a \bar{a} b \bar{b}}$ has precisely the same tensor structure as the pure gravity amplitude computed in [11].

Next, let us consider diagrams with only hard fermion propagators. For the most part these diagrams are the same as in the spin $\leq 1$ case. The only subtlety is that the propagator for a spin $\frac{3}{2}$ fermion is different from that of a spin $\frac{1}{2}$ fermion. The kinetic term for the gravitino fluctuation is:

$$
\mathcal{L}_{\frac{3}{2}}=\frac{i}{12} \bar{\lambda}_{\rho} \gamma^{\rho \sigma \mu} \nabla_{\mu} \lambda_{\sigma}
$$

However, by choosing a gauge $\gamma^{\mu} \lambda_{\mu}=0$ and anti-commuting gamma matrices, we obtain

$$
\mathcal{L}_{\frac{3}{2}}=-\frac{i}{2} \bar{\lambda}_{\mu} \not \nabla \lambda^{\mu}
$$

which is simply four copies of a spin $\frac{1}{2}$ fermion. Notice the manifest spin Lorentz symmetry acting on gravitino index!

Like before, the leading in $z$ contribution comes from taking a factor of $\not$ from every fermion propagator numerator. Applying the same arguments as in the gauge theory case, the contribution from diagrams with only hard fermion propagators becomes

$$
\begin{aligned}
\delta \mathcal{M}_{2}^{a \bar{a} b \bar{b}}=D^{[a b] \bar{a} \bar{b}}+\bar{D}^{[\bar{a} \bar{b}] a b} & +q^{(a} E^{b) \bar{a} \bar{b}}-\eta^{a b} q_{c} E^{c \bar{a} \bar{b}} \\
& +q^{\left(\bar{a} \bar{E}^{\bar{b}) a b}\right.}-\eta^{\bar{a} \bar{b}} q_{\bar{c}} \bar{E}^{\bar{c} a b}
\end{aligned}
$$

which is very similar to the corresponding expression in the gauge theory (see equation (3.10)) except that there are two additional indices. 
Summing contributions from all diagrams with no hard propagators and all diagrams with only hard fermion propagators, we obtain the amplitude

$$
\begin{aligned}
\mathcal{M}^{a \bar{a} b \bar{b}}= & \mathcal{M}_{\mathrm{grav}}^{a \bar{a} b \bar{b}}+\delta \mathcal{M}_{1}^{a \bar{a} b \bar{b}}+\delta \mathcal{M}_{2}^{a \bar{a} b \bar{b}} \\
= & \mathcal{M}_{\mathrm{grav}}^{a \bar{a} b \bar{b}}+A^{a b} \eta^{\bar{a} \bar{b}}+\bar{A}^{\bar{a} \bar{b}} \eta^{a b}+B^{[a b][\bar{a} \bar{b}]}+z\left(C^{[a b]} \eta^{\bar{a} \bar{b}}+\bar{C}^{[\bar{a} \bar{b}]} \eta^{a b}\right) \\
& +D^{[a b] \bar{a} \bar{b}}+\bar{D}^{[\bar{a} \bar{b}] a b}+q^{(a} E^{b) \bar{a} \bar{b}}-\eta^{a b} q_{c} E^{c \bar{a} \bar{b}}+q^{(\bar{a}} \bar{E}^{\bar{b}) a b}-\eta^{\bar{a} \bar{b}} q_{\bar{c}} \bar{E}^{\bar{c} a b}+\mathcal{O}(1 / z)
\end{aligned}
$$

where $\mathcal{M}_{\text {grav }}^{a \bar{a} b \bar{b}}$ is the contribution from pure gravity considered in [11]. Without loss of generality, we take graviton 1 to have negative helicity and graviton 2 to be arbitrary. The graviton polarizations are symmetric, traceless products of gauge polarizations, so they take the form

$$
\begin{aligned}
& \epsilon_{1 a \bar{a}}^{-\bar{a}}=q_{a} q_{\bar{a}} \stackrel{\text { gauge }}{=} p_{1 a} p_{1 \bar{a}} / z^{2} \\
& \epsilon_{2 a \bar{a}}^{ \pm \pm}=\left\{\begin{array}{cc}
q_{a} q_{\bar{a}}, & (++) \\
\left(q_{a}^{*}+z p_{1 a}\right)\left(q_{\bar{a}}^{*}+z p_{1 \bar{a}}\right), & (--)
\end{array}\right.
\end{aligned}
$$

Using equation (4.20), we find that the $(--,++),(--,--)$, and $(--, T)$ amplitudes go as:

$$
\begin{aligned}
\mathcal{M}^{--,++} & =q_{a} q_{\bar{a}} \mathcal{M}^{a \bar{a} b \bar{b}} q_{b} q_{\bar{b}} \\
& \rightarrow \mathcal{O}(1 / z) \\
\mathcal{M}^{--,--} & =\frac{1}{z^{2}} p_{1 a} p_{1 \bar{a}} \mathcal{M}^{a \bar{a} b \bar{b}}\left(q_{b}^{*}+z p_{1 b}\right)\left(q_{\bar{b}}^{*}+z p_{1 \bar{b}}\right) \\
& \rightarrow \mathcal{O}(1 / z) \\
\mathcal{M}^{--, T} & =\frac{1}{z^{2}} p_{1 a} p_{1 \bar{a}} \mathcal{M}^{a \bar{a} b \bar{b}} \epsilon_{b}^{T} \epsilon_{\bar{b}}^{T} \\
& \rightarrow \mathcal{O}\left(1 / z^{2}\right)
\end{aligned}
$$

Thus, we have shown all amplitudes with at least two gravitons vanish at large $z$.

\subsection{Mixed graviton amplitudes}

In theories of (super)gravity coupled to scalars and fermions, any tree amplitude with at least two gravitons obeys a recursion relation. However, in analogy with the gauge case, gravitational theories also admit recursion relations for amplitudes with only one graviton. Our argument parallels that of the spin $\leq 1$ theory. Again, by fixing light-cone gauge for the background and an $R_{\xi}$ gauge for the fluctuation we can remove all $\mathcal{O}\left(z^{2}\right)$ and $\mathcal{O}(z)$ interactions. Then we just have to check explicit diagrams in which particle 1 is a graviton and particle 2 is not.

First, let us consider diagrams with only hard fermion propagators. No matter the identity of particle 2 , these diagrams take the form

$$
\mathcal{M}^{a \cdots} \sim \gamma^{a} \not 1 \ldots
$$

where $a$ is a graviton index. This has to be true because particle 1 connects to two fermion lines, and gravitons can only couple to fermions in a very specific way. Finally, dotting 
$\mathcal{M}^{a \ldots}$ into a negative helicity graviton polarization $\epsilon_{1 a \bar{a}}^{--}=q_{a} q_{\bar{a}}$, we see that this leading in $z$ contribution vanishes.

Diagrams with no hard propagators come from the term $h_{\mu \nu} T_{(1)}^{\mu \nu}$, which naively includes interactions involving derivatives of hard matter fields. However, the deDonder $R_{\xi}$ gauge removes such terms. Consequently, the interaction vertex goes at most as $\mathcal{O}(1)$. Next, dotting the mixed diagram into the graviton polarization $\epsilon_{1 a \bar{a}}^{--}=p_{a} p_{\bar{a}} / z^{2}$, we realize that if particle 2 is a scalar, fermion, or gluon, then its polarization goes at most as $z$ and so this contribution to the amplitude vanishes at large $z$. Finally, by explicitly checking the diagram where particle 2 is a gravitino, we find that all of the mixed diagrams vanish at large $z$. This completes our proof that recursion relations hold for any amplitude with at least one graviton.

\section{Conclusion}

Recursion relations are a generic feature of tree amplitudes that vanish at large complexified momentum. In this paper we show that this criterion holds for a broad class of amplitudes in two derivative gauge and (super)gravity theories in any number of dimensions. In particular, for a theory of spin $\leq 1$, any amplitude with at least one gluon can be recursed; for a theory of spin $\leq 2$ this is true of any amplitude with at least one graviton. Said another way, recursion relations hold as long there is at least one external leg with the highest spin possible. This is sensible because a higher spin particle enjoys a greater gauge redundancy that is essential for obtaining nice large $z$ behavior in an amplitude. In particular, only by choosing light-cone gauge for the backgrounds and an additional $R_{\xi}$ gauge for the fluctuations were we able to eliminate large $z$ contributions. Moreover, the $R_{\xi}$ gauge was especially critical for proving vanishing large $z$ behavior in amplitudes with only one gluon or only one graviton.

Given the existence of a broad new class of on-shell recursion relations, it is reasonable to ask whether any of these tools may be used to generate new closed form expressions for amplitudes in generic field theories. Unfortunately, since these generic recursion relations require the presence of a least one external leg with the highest spin possible, any sequence of recursions invariably terminates at an amplitude whose external legs are not of maximal spin. For instance, consider an on-shell amplitude in QCD with only external gluons. Applying on-shell recursion relations, one finds that the recursion ultimately stalls once all the lower point amplitudes involve only external fermions. That said, the possibility of deriving new closed form expressions is certainly an intriguing one, and requires further study.

Finally, we remark on the interesting fact that the spin $\leq 2$ amplitude (equation (4.20)) has precisely the same structure as the square of the spin $\leq 1$ amplitude (equation (3.11)). This is a non-trivial consistency check against the famous KLT relation [14] that equates closed string tree amplitudes with sums over products of open string tree amplitudes. At low energies this statement persists as a relation between amplitudes in Yang-Mills and gravity, and in fact for the case of MHV, new formulas for the the KLT relations have been derived directly from the BCFW recursion relations [15]. Since the KLT relations also 
relate amplitudes in $\mathcal{N}=4 \mathrm{SYM}$ and $\mathcal{N}=8$ supergravity (a subset of the theories under consideration in this paper), we should expect such a relation between our expressions for spin $\leq 1$ and spin $\leq 2$ amplitudes.

\section{Acknowledgments}

It is a pleasure to thank N. Arkani-Hamed, H. Elvang, D. Freedman, and J. Kaplan for very helpful physics discussions and comments on the manuscript. CC is supported in part by DOE grant DE-FG02-91ER40654.

\section{A Exact expressions for $X^{\mu \nu \rho \sigma}$ and $Y^{\lambda \mu \nu \rho \sigma}$}

In this section we write down some exact expressions for $X^{\mu \nu \rho \sigma}$ and $Y^{\lambda \mu \nu \rho \sigma}$. First, let us parse these tensors according to the spin of the field contributing: $s=0, \frac{1}{2}, 2$. An explicit calculation shows [16]:

$$
\begin{aligned}
X_{s=0}^{\mu \nu \rho \sigma} & =\eta^{\mu \rho} \partial^{\nu} \Phi \partial^{\sigma} \Phi-\frac{1}{2} \eta^{\mu \nu} \partial^{\rho} \Phi \partial^{\sigma} \Phi-\frac{1}{4} \eta^{\mu \rho} \eta^{\nu \sigma} \partial_{\lambda} \Phi \partial^{\lambda} \Phi+\frac{1}{8} \eta^{\mu \nu} \eta^{\rho \sigma} \partial_{\lambda} \Phi \partial^{\lambda} \Phi \\
X_{s=\frac{1}{2}}^{\mu \nu \rho \sigma} & =\frac{3}{8} \eta^{\mu \rho} \bar{\Psi} i \gamma^{\nu} \widehat{\nabla}^{\sigma} \Psi-\frac{1}{4} \eta^{\mu \nu} \bar{\Psi} i \gamma^{\rho} \widehat{\nabla}^{\sigma} \Psi-\frac{1}{4} \eta^{\mu \rho} \eta^{\nu \sigma} \bar{\Psi} i \widehat{\chi} \Psi+\frac{1}{8} \eta^{\mu \nu} \eta^{\rho \sigma} \bar{\Psi} i \widehat{\not} \Psi \\
X_{s=2}^{\mu \nu \rho \sigma} & =-R^{\mu \rho \nu \sigma}+2 \eta^{\mu \rho} R^{\nu \sigma}+\eta^{\mu \nu} R^{\rho \sigma}+\frac{1}{2} \eta^{\mu \rho} \eta^{\nu \sigma} R-\frac{1}{4} \eta^{\mu \nu} \eta^{\rho \sigma} R \\
Y_{s=0}^{\lambda \mu \nu \rho \sigma} & =0 \\
Y_{s=\frac{1}{2}}^{\lambda \mu \nu \rho \sigma} & =\frac{1}{48} g^{\nu \sigma} \bar{\Psi} \gamma^{\mu \rho \lambda} \Psi \\
Y_{s=2}^{\lambda \mu \nu \rho \sigma} & =0
\end{aligned}
$$

where $\bar{\Psi} \widehat{\nabla}_{\mu} \Psi=\bar{\Psi} \nabla_{\mu} \Psi-\nabla_{\mu} \bar{\Psi} \Psi$ and we have only included contributions from the kinetic terms. Aside from terms that couple to the trace of the graviton (which are removed by the dilaton field redefinition) these expressions match the general structure deduced in equations (4.13) and (4.15).

Open Access. This article is distributed under the terms of the Creative Commons Attribution Noncommercial License which permits any noncommercial use, distribution, and reproduction in any medium, provided the original author(s) and source are credited.

\section{References}

[1] S.J. Parke and T.R. Taylor, An Amplitude for $n$ Gluon Scattering, Phys. Rev. Lett. 56 (1986) 2459 [SPIRES].

[2] E. Witten, Perturbative gauge theory as a string theory in twistor space, Commun. Math. Phys. 252 (2004) 189 [hep-th/0312171] [SPIRES].

[3] F. Cachazo, P. Svrček and E. Witten, MHV vertices and tree amplitudes in gauge theory, JHEP 09 (2004) 006 [hep-th/0403047] [SPIRES]. 
[4] R. Britto, F. Cachazo and B. Feng, New Recursion Relations for Tree Amplitudes of Gluons, Nucl. Phys. B 715 (2005) 499 [hep-th/0412308] [SPIRES].

[5] R. Britto, F. Cachazo, B. Feng and E. Witten, Direct Proof Of Tree-Level Recursion Relation In Yang-Mills Theory, Phys. Rev. Lett. 94 (2005) 181602 [hep-th/0501052] [SPIRES].

[6] S.D. Badger, E.W.N. Glover, V.V. Khoze and P. Svrček, Recursion Relations for Gauge Theory Amplitudes with Massive Particles, JHEP 07 (2005) 025 [hep-th/0504159] [SPIRES].

[7] F. Cachazo and P. Svrček, Tree level recursion relations in general relativity, hep-th/0502160 [SPIRES].

[8] J. Bedford, A. Brandhuber, B.J. Spence and G. Travaglini, A recursion relation for gravity amplitudes, Nucl. Phys. B 721 (2005) 98 [hep-th/0502146] [SPIRES].

[9] N.E.J. Bjerrum-Bohr, D.C. Dunbar, H. Ita, W.B. Perkins and K. Risager, $M H V$-vertices for gravity amplitudes, JHEP 01 (2006) 009 [hep-th/0509016] [SPIRES].

[10] P. Benincasa, C. Boucher-Veronneau and F. Cachazo, Taming tree amplitudes in general relativity, JHEP 11 (2007) 057 [hep-th/0702032] [SPIRES].

[11] N. Arkani-Hamed and J. Kaplan, On Tree Amplitudes in Gauge Theory and Gravity, JHEP 04 (2008) 076 [arXiv:0801.2385] [SPIRES].

[12] G. 't Hooft and M.J.G. Veltman, One loop divergencies in the theory of gravitation, Annales Poincare Phys. Theor. A 20 (1974) 69 [SPIRES].

[13] Z. Bern and A.K. Grant, Perturbative gravity from QCD amplitudes, Phys. Lett. B 457 (1999) 23 [hep-th/9904026] [SPIRES].

[14] H. Kawai, D.C. Lewellen and S.H.H. Tye, A Relation Between Tree Amplitudes of Closed and Open Strings, Nucl. Phys. B 269 (1986) 1 [SPIRES].

[15] H. Elvang and D.Z. Freedman, Note on graviton MHV amplitudes, JHEP 05 (2008) 096 [arXiv:0710.1270] [SPIRES].

[16] N.E.J. Bjerrum-Bohr, Quantum gravity, effective fields and string theory, hep-th/0410097 [SPIRES]. 